\title{
Resist or Give in to an Alternative: Post-Decisional Evaluations of Cost, Value and Regret in the Choice
}

\author{
Pierluigi Diotaiuti, Giuseppe Valente, Stefania Mancone, Angela Grambone \\ Department of Human Sciences, Society and Health, University of Cassino and Southern Lazio, Cassino, Italy \\ Email: p.diotaiuti@unicas.it
}

How to cite this paper: Diotaiuti, P., Valente, G., Mancone, S., \& Grambone, A. (2020). Resist or Give in to an Alternative: Post-Decisional Evaluations of Cost, Value and Regret in the Choice. Psychology, 11, 245-267.

https://doi.org/10.4236/psych.2020.112016

Received: December 19, 2019

Accepted: February 7, 2020

Published: February 10, 2020

Copyright ( 2020 by author(s) and Scientific Research Publishing Inc. This work is licensed under the Creative Commons Attribution International License (CC BY 4.0).

http://creativecommons.org/licenses/by/4.0/

\begin{abstract}
This study presents a comparative analysis of post-decisional evaluations with reference to a choice made by the subject between an option with an antecedent that had a pleasurable finality while the alternative option was orientated respectively to the undertaking something useful, to fulfilling an obligation, or to the respect of a prior commitment with others. The study focalizes on a particular condition of the subject's awareness; that is to say that the decision was made with a will to resist to the alternative option, or the knowledge that he has substantially chosen to succumb to the pressure exercised by the alternative option. The study showed such an awareness condition had a significant effect on fundamental judgments that accompany and follow the decisional act, i.e. the rating of the cost of the choice, the value attributed to the choice, the regret associated to the option renounced by the subject.
\end{abstract}

\section{Keywords}

Post-Decisional Evaluation, Dissonance, Regret, Decisional Cost, Value of Choice, Temptation

\section{Introduction}

Much of the past literature on decision making has focused on the condition of a subject faced with an evaluation of substantially homogeneous options (two or more consumer products, holiday destinations, a choice of postcards, the chance to earn money, etc.) (Cohen \& Goldberg, 1970; Hui, \& Molden, 2014; Sharot et al. 2009). The risk of the effects of cognitive overlap in situations of choice where the options have common attributes has already been investigated by several academics (Trump \& Brucks, 2012; Brehm \& Cohen, 1962), who state that the 
cognitive overlap, both real or imagined, reduces the cognitive dissonance linked to the choice made, and therefore, lowers the level of difficulty and decisional cost for the subjects. Of course, the conceptual reference of this line of studies is the Model for Cognitive Dissonance (Festinger, 1957; 1964) regained and re-articulated in terms of mental fatigue also by Swait \& Adamowicz, 2001; Basu \& Savani, 2017, and Jonker et al., 2018. In the light of these observations, it would seem remarkable to give space to research that investigates human decisional behaviour from a more naturalistic point of view and linked to the dynamics of the different contexts in which the subject happens to find himself in (Klein, 1998). From this point of view, it is more likely that the subject be encouraged towards an orientation between options that are substantially not equivalent (Friedman et al., 2018). For example, deciding to spend an evening with friends rather than getting down to finishing an urgent job; choosing to read a book in perfect relaxation rather than doing a work out in the gym, etc. This naturally follows the considerations already described by Johnson (1984) as he stressed the need to move from a hierarchical model of choice, related to the evaluation of "comparable alternatives" (for reference to the same attributes or qualities) to use valuation strategies implemented in relation to what he termed "un-comparable alternatives" (where comparability is the degree to which alternatives are described or represented by the same attributes) (see also Bettman et al., 1998). More recently, however, construal level theory (CLT) with a purely cognitive orientation has risen to the forefront, focusing on the level of construals that receive greater attention or weight in evaluation (see Dhar \& Kim, 2007; Trope \& Liberman, 2010). An emerging trend in this literature has been shifting from the consideration of single goal and isolated choice settings to scenarios where people pursue multiple goals through a sequence of choices. For example, Fishbach and Dhar (2005) found that when in the presence of multiple competing goals, people can either opt to behave in ways that are consistent with a goal or behave in ways that disengage from one goal in favor of an alternate goal. It depends on whether an initial action is interpreted in terms of goal commitment or goal progress. An interesting question is regarding the set of factors that can determine whether a specific action is interpreted in terms of goal progress or goal commitment (Fishbach et al., 2006). The study we are presenting here is focused on the condition in which the subject has previously formulated his intention (first option) and then subsequently re-evaluated his choice in the light of a new stimulus/temptation (second option). For example, the subject intended to rest but was then encouraged to go out with a friend. This situation could be related to the Model of Pre-decisional Conflict as illustrated by Costanzo (2013), who underlines its influence on the decisions of consumer purchasing. From a post-decision perspective we believe that it may be appropriate to underline that the subject's decision could evolve under two different psychological conditions, both influential in a different way. The first condition could be defined as the subject's resistance, in that he performs his de- 
cisional act with an attitude of "resistance": when evaluating the options he tests the first hypothesis by comparing its validity with the second option. The second condition could be defined as the subject's exposition to succumb. In this case he accompanies the decisional act with an initial attitude of willingness towards the stimuli/encouragement emanating from the option: the subject does not try to evade the influence emanating from the option. It is also useful to distinguish the evaluation that the subject makes after having completed his decisional act. On this level it would be feasible for the subject to consider if the result of the decision was due to an act of resistance or succumbing to a temptation/option. In the literature the question of resistance in choosing has been mostly associated to the question of temptation, potentially more attractive and more remunerative than the first option. Resisting or failing, in this sense, therefore succumbing, calls upon certain aspects linked to the control of impulses, inhibition skills, attention control, the regulation of affections (Baumeister \& Heatherton, 1996; Metcalfe \& Mischel, 1999; Hoffmann \& Trautmann, 2008; Ent et Baumeister, 2014). In the case of temptation we are presented with two opposition strategies: resist when faced directly with the source of the temptation or deliberately try to avoid contact. In our study the antecedent is pleasurable for the subject while the options can be either equivalent or structurally different. In the first case (the equally pleasurable option) there will obviously be less dissonance than the other options. The innovative aspect of our contribution is in the evaluation of how the subject's final judgment changes when an originally hedonistic and intrinsically rewarding position (intention) becomes questionable. Once the decisional action is concluded, both in the case where the consequences of the course of action undertaken are immediately clear to the subject, and in the case where these are only present in terms of expectations, then the final area of post-decisional evaluation comes into play (Svenson et al., 2009; Salo \& Svenson, 2001; Svenson, 2006; Krosch et al., 2012). The prevalent literature on this subject has investigated above all a level of evaluation associated with the feeling of regret, that is to say, the level of regret/remorse that follows the decision taken and associated with thinking about the option not taken or which has been renounced (Kahneman, 1994; Zeelenberg \& Beattie, 1997; Gilbert et al., 2004; Van Dijk \& Zeelenberg, 2005; Zeelenberg \& Pieters, 2007; Seta et al., 2008; Beike et al., 2009; Raeva et al., 2010; Marcatto \& Ferrante, 2012; Wilson \& Gilbert, 2005).

A second component of post-decisional evaluation relative to the estimate of the decisional cost (indicated in literature prevalently as cognitive effort) for the subject, who has to orientate his intentional and behavioural route starting from a specific contextual condition and structured in accordance with a specific level of structural dissonance (pleasure/pleasure; pleasure/utility; pleasure/obligation; pleasure/commitment). I.e. whereas the first option is alternately confronted with a condition considered equally pleasant, or useful, or perceived as an obligation, or related to commitments undertaken with other people. As reported by 
Park et al. (2015), previous decision making literature defined cognitive effort as an internal information search effort, such as the use of working memory or performing mental calculation without any external aid. For example, one common way to manipulate cognitive effort is to vary the size of the choice set in a decision task (e.g., Iyengar \& Lepper, 2000; Sagi \& Friedland, 2007). Further studies proposed to use a broader definition of cognitive effort as the total cognitive resources used in the decision process (e.g., Russo \& Dosher, 1983), which may be derived from both internal and external information search and overall time spent (e.g., Garbarino \& Edell, 1997). Moreover an articulated model of post-decisional evaluation cannot neglect to consider a third factor: an estimate of the value attributed by the subject to the decision made (Tversky \& Kahneman, 1981; Kahneman et al., 1990; Shamoun \& Svenson, 2002; Schwartz et al., 2002; Higgins, 2006; Hsee \& Rottenstreich, 2004). In the literature, the value of the choice is based prevalently on an evaluation of the total balance of the "utility" of the attributes associated to the single options (vd. Multi-Attribute Utility Theory, MAUT; Adams \& Fagot, 1959; Edwards et al., 1975; Keeney \& Raiffa, 1976; Hammond et al., 1999). Mellers \& Cooke (1994) had already illustrated how the evaluation of the value is a dynamic and not a rigid process, so even minimal changes of perspective can influence judgment and therefore determine fluctuations in the rating of values/utility attributed to the single or group predicates.

For the purposes of the present work, we have mainly referred to Fishbach and Dhar's Construal Level Theory (CLT, 2005) considering scenarios where people pursue multiple goals through a sequence of choices. We assumed that it was for the most likely and frequent subjects in everyday life the condition of choosing between paths of action related to different domains/goals (for example, preferring something pleasant over obtaining a utility, or giving up pleasure in the name of a higher duty or not to disappoint the expectations of a reference group). The first hypothesis was therefore for us to verify to what extent the goal of an alternative action could influence the judgments of cost, value and regret inherent in the chosen course of action. A further consideration guided the construction of the study, namely that in a dynamic decision-making process the evaluation of alternatives is not always simultaneous and parallel, but often temporally disjointed, so it is plausible to believe that at first the subject formulates a certain intention of choice, on the basis of certain pressures and context assessments, and that in a second moment, a change of perspective or a specific external induction pushes him to question the first formulation of intention, and to reach a final decision that reaffirms the initial will or modifies the outcome in favor of the new alternative.

Our second hypothesis was therefore that post-decisional evaluation of a choice between alternatives may also be affected by a specific level of awareness of the subject, i.e. recognizing that in the end they have resisted the alternative temptation/induction or have "given in" and had to give up the first intention. 
So in synthesis the main research objective of our study was to understand whether post-decisional judgments (i.e., the cost of the decision, the estimation of the value attributed to the choice, and the regret associated with the waived option) would vary significantly, within contexts that held a second option in (goal) dissonance with the original intention of the subject, considering also the special condition of awareness that may accompany the decision-making process between competing alternatives, i.e., the awareness of having eventually resisted the alternative or having succumbed to it.

\section{Methods}

\subsection{Participants}

201 university Students with average age $=22.90$; DS $=3.23$; Males $=93(46.3 \%)$; Females $=108(53.7 \%)$ have been randomly selected from among all those enrolled and attending the courses of Economics, Philosophy, Communication and Education (total students number: 420). The sample size determination was made by setting a 1 -alpha confidence level at $95 \%$, therefore with $\mathrm{z}$ normal value at the confidence level of 1.96 . The following two formulas were applied, where the second operated the correction for small populations (<20,000): 1) $X_{o}=Z^{2}$ ( $p$ $\left.{ }^{*} q\right) / b^{2}$, with $p$ as the proportion to be estimated and $q$ the proportion of complementary character and $b$ the desired precision set at 5\%. Hence: $3.842(0.46 \times$ $0.54) / 0.0025=381.72$; 2$) X=X_{o} /\left[1+\left(X_{o} /\right.\right.$ pop $\left.)\right]$, with pop the numerical value of the reference population. Hence: $381.72 /[1+(381.72 / 420)]=200$. Tools administration took place upon release and signing of the form for an informed consent of participation in accordance with the Declaration of Helsinki.

\subsection{Procedure and Instruments}

The study protocol envisaged proposing to the participants in succession four scenarios, in which a choice of action aimed at obtaining a pleasant condition was presented, subsequently questioned by a second alternative, respectively equally pleasant in the outcome (first scenario), aimed at achieving a utility (second scenario), aimed at fulfilling an obligation (third scenario), aimed at meeting the expectations of a group (fourth scenario). Decision-making scenarios reproducing situations from everyday life were defined through a preliminary processing and selection procedure conducted by a group of 50 undergraduate students who first identified 15 action choices aimed to be pleasant, 8 aimed to be useful, 9 perceived as an obligation, 10 related to commitments undertaken with other people. Subsequently, at each situation, students rated a score from 1 to 7 to indicate the degree of representativeness of each specific condition. Considering the higher scores obtained, two conditions of pleasure, one of utility, one of obligation and one of commitment were ultimately selected for the protocol. Table 1 shows the sequence of the four goal comparisons presented through the scenarios. 
Table 1. Competing action objectives in the identification scenarios.

\begin{tabular}{ccc}
\hline Table 1 & First Action Choice & Second Action Choice \\
\hline First Scenario & Pleasant Goal & Pleasant goal \\
Second Scenario & Pleasant Goal & Useful Goal \\
Third Scenario & Pleasant Goal & Goal of Obligation \\
Fourth Scenario & Pleasant Goal & Goal of Compliance \\
& & with Expectations \\
\hline
\end{tabular}

In the first scenario the pleasurable condition was confronted with an alternative option of equal valence: the temptation even presented the same kind of pleasurable outcome should the subjects decide to choose it: "You intend to go for a relaxing, late afternoon walk. You receive a phone call from a friend who informs you that at exactly that time there will be your favorite film on $T V$ '. In the second scenario the pleasurable condition was confronted with an alternative whose outcome would lead to a useful acquisition for the subjects: "You realize that the fridge is empty and tomorrow the shops will be shut because it's a bank holiday". In the third scenario the subjects had to decide between the pleasurable condition and an alternative linked to an obligation: "Your mother reminds you that you have to tidy your room in the afternoon because there are guests arriving for dinner". Finally, in the fourth scenario the subjects were invited to confront with an alternative option associated to a previously made commitment in favor of other subjects: "You remember that you committed yourself to preparing a report for a group project to hand in to your professor the following day".

Each scenario presented two possible outcomes: 1) Resisting the alternative option while remaining firm with the first program (pleasant situation); 2) Succumbing to the pressure of the alternative.

Subjects were asked to read each scenario as far as possible identifying consecutively with both expected outcomes (resist and succumb to the alternative). Later on, for each single condition (resist/succumb) and scenario the subjects were invited to express their post-decisional evaluations articulated in three items using a five point Likert Scale. The first item asked the subject to express an evaluation of the "cost" attributed to the decision made, or rather to the estimated effort needed to resist or succumb to the option. The second item asked the subject to give a rating in terms of the "subjective value" attributed to the choice made in the two conditions. The third item asked the subject to indicate a measure of "regret" or distress, intended as persistent thinking about the non chosen option.

For ex.

A (resist): You intend to go for a relaxing, late afternoon walk but you remember that you committed yourself to preparing a report for a group project to hand in to your professor the following day. Despite the prior commitment you don't change your mind.

- From 1 to 5, how much would it have cost you to maintain your initial inten- 
tion?

- What value from 1 to 5 would you have attributed to not changing your mind?

- After your choice, from 1 to 5, how long would you have kept thinking about the alternative option?

B (succumb): You intend to go for a relaxing, late afternoon walk but you remember that you committed yourself to preparing a report for a group project to hand in to your professor the following day. You change your mind and decide to keep your prior commitment so as not to disappoint your group.

- From 1 to 5, how much would it have cost you to modify your initial intention?

- What value from 1 to 5 would you have attributed to the fact that you changed your mind?

- After your choice, from 1 to 5, how long would you have kept thinking about the alternative option?

\section{Statistical Analysis}

The data were processed using the statistical software SPSS version 22. Since the objective was to measure the change in the estimates of cost, value and regret (dependent variables) after the sequential administration of the four identification scenarios, within-subjects Repeated Measures Anova were carried out using the Generalized Linear Model procedure. The analyses were carried out separately for the resistance and succumbing versions provided for by the protocol. The four scenarios were then inserted as stimulus factor levels within the subjects. The average of the estimate of cost, value and regret, associated with the choice with which the participants made the identification, represented the dependent variables analyzed. Assumptions considered for Repeated Measures ANOVA were Normality (the test variables follow a multivariate normal distribution in the population) and Sphericity (the variances of all difference scores among the test variables must be equal in the population). If $p>0.05$ at Mauchley's test, Sphericity could be assumed. Where the assumption was not met, has been used the p-value from the Greenhouse-Geisser correction row. The comparison of marginal means was made using Sidak's correction method. Pearson $r$ correlation and Omega-squared $\left(\omega^{2}\right)$ have been used as values of the effect size. According to Cohen $(1988,1992)$, for Pearson's $r$ the effect size is low if the value of $\mathrm{r}$ varies around 0.1 , medium if $\mathrm{r}$ varies around 0.3 , and large if $\mathrm{r}$ varies more than 0.5; while Field (2013) suggested for Omega Squared the following interpretation heuristics: 0 - 0.01: a very small effect; 0.01 - 0.06: small effect; 0.06 - 0.14: medium effect; > 0.14: large effect.

\section{Results}

Table 2 reports the main descriptive statistics of dependent variables (Value, Cost, Regret) differentiated according to the decision-making scenario and the identification condition (resistance and succumbing). 
Table 2. Descriptive statistics of dependent variables.

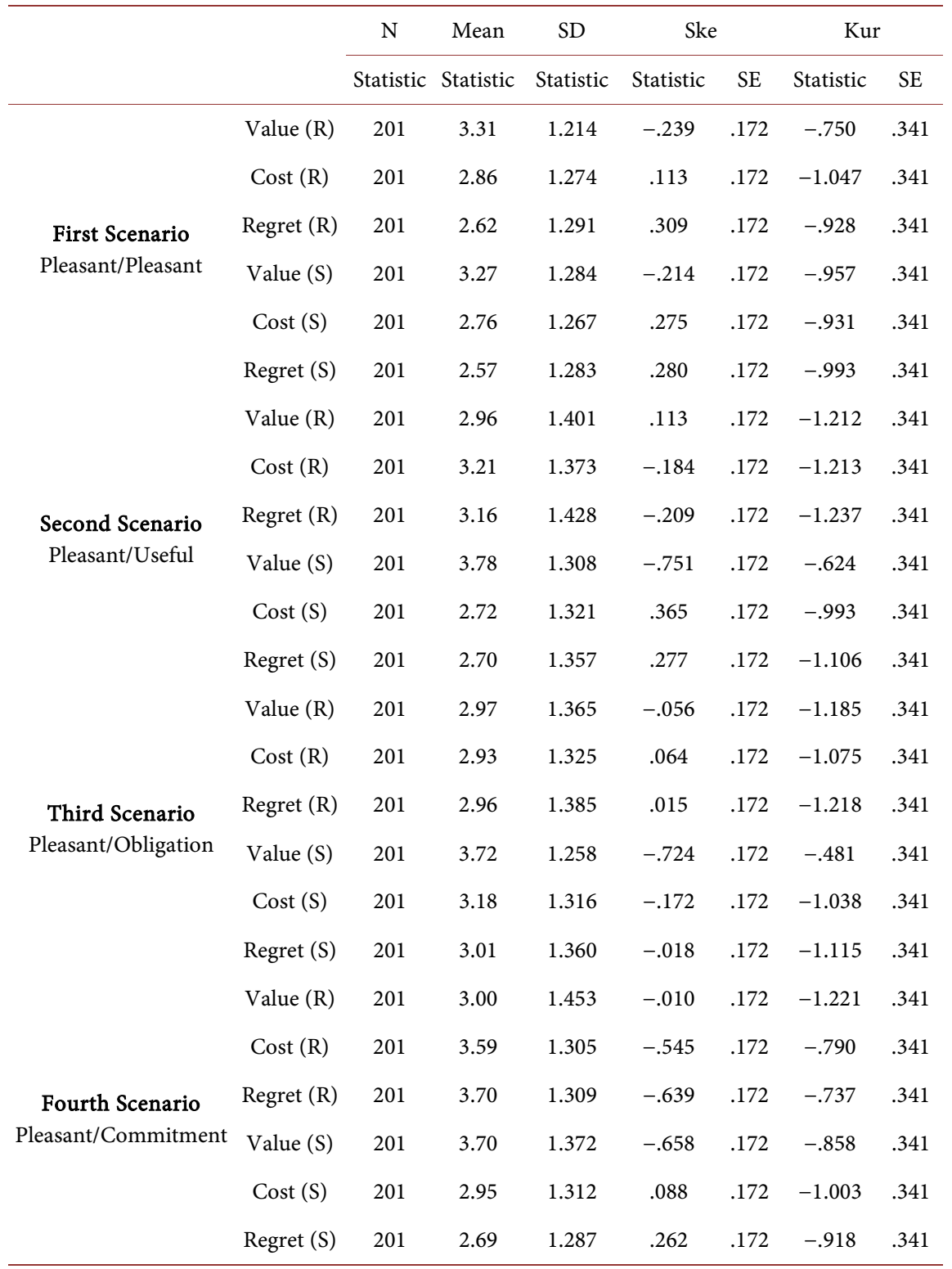

Legend: $\mathrm{R}=$ Condition of Resistance; $\mathrm{S}=$ Condition of Succumbing; $\mathrm{SD}=$ Standard Deviation; $\mathrm{SE}=\mathrm{Stan}-$ dard Error; Ske = Skewness; $\mathrm{Ku}=$ Kurtosis.

Regarding the normality assumption, values showed some kurtosis but nothing too alarming $(<1.3)$. The preliminary verifications of the assumptions excluded the presence of multivariate outliers. Mardia's multivariate kurtosis index (23.56) was in fact below the critical value $p(p+2)=24$; so the relationship between the variables can be considered substantially linear. Low co-linearity was indicated by the low VIF values (Variance Inflaction Factor) $<2$ and high tolerance values $>.60$. For verification of the assumptions on the residuals, the average between the standardized and raw residuals was equal to 0 ; the Durbin-Watson test had a value of 1.94 and was therefore indicative of the absence of autocorrelation. 
First was evaluated the cost factor of the decision for the four levels (scenarios) in the condition of resistance. Given that Mauchly's test was not significant (Sig. .124), the condition for sphericity was verified, $\chi^{2}(5)=1.698, p=.889$. The effect of the repeated measurements was significant. $\mathrm{F}(3,600)=15.455 ; p=.000$; $\mathrm{r}=.27 ; \omega^{2}=.04$. Has been then compared the marginal averages with the Sidak correction method (see Figure 1).

From the analysis emerged that estimated mean of cost when the alternative option was also a pleasurable choice resulted significantly lower than both the choices when the alternative was an option of utility for the subject $(p=.024)$ and when was an option concerning a prior commitment with others $(p=.000)$ (see Table 3). When the resistance in a pleasurable choice was compared to a utility option, the cost of this resistance was significantly lower when the alternative is a prior commitment with others $(p=.007)$. When the resistance in the pleasurable choice was compared with an option of obligation for the subject, the cost of the resistance was significantly lower than the situation in which the alternative was a prior commitment with others $(p=.000)$. The cost of resistance was maximum when the alternative option was a prior commitment and was significantly high when it presented characteristics of utility for the subject. The cost of the resistance was lower when the alternative was equally pleasurable or when it presented an obligation for the subject. In order to improve the comparison was also performed a planned comparison adopting the reverse Helmert Contrast method (Difference) concentrating on the resistance to a prior commitment (level four). The comparison with the other levels presented a significant $\mathrm{F}$ test: $\mathrm{F}(1,200)=38.994$ with $p=.000$, verifying power equal to 1.000 (computed using alpha $=.05$ ) and an $r$-effect size equal to .40 .

Table 3. Pairwise Comparison of Cost in condition of Resistance.

\begin{tabular}{|c|c|c|c|c|c|}
\hline (I) & $(\mathrm{J})$ & MD & SE & $p$ & $\mathrm{BCa} 95 \% \mathrm{CI}$ \\
\hline \multirow{3}{*}{1} & 2 & $-.353^{*}$ & .121 & .024 & {$[-.68,-.03]$} \\
\hline & 3 & -.075 & .125 & .992 & {$[-.41, .26]$} \\
\hline & 4 & $-.736^{*}$ & .117 & .000 & {$[-1.05,-.42]$} \\
\hline \multirow{3}{*}{2} & 1 & $.353^{*}$ & .121 & .024 & {$[.11, .59]$} \\
\hline & 3 & .279 & .118 & .112 & {$[.04, .51]$} \\
\hline & 4 & $-.383^{*}$ & .117 & .007 & {$[-.61,-.15]$} \\
\hline \multirow{3}{*}{3} & 1 & .075 & .125 & .992 & {$[-.26, .41]$} \\
\hline & 2 & -.279 & .118 & .112 & {$[-.59, .04]$} \\
\hline & 4 & $-.662^{*}$ & .121 & .000 & {$[-.98,-.34]$} \\
\hline \multirow{3}{*}{4} & 1 & $.736^{*}$ & .117 & .000 & {$[.42,1 \quad .05]$} \\
\hline & 2 & $.383^{*}$ & .117 & .007 & {$[.07, .69]$} \\
\hline & 3 & $.662^{*}$ & .121 & .000 & {$[.34, .98]$} \\
\hline
\end{tabular}

Table legend: $\mathrm{MD}=$ difference of means; $\mathrm{SE}=$ standard error; $\mathrm{CI}=$ confidence interval; $\mathrm{ps}<.05$. 


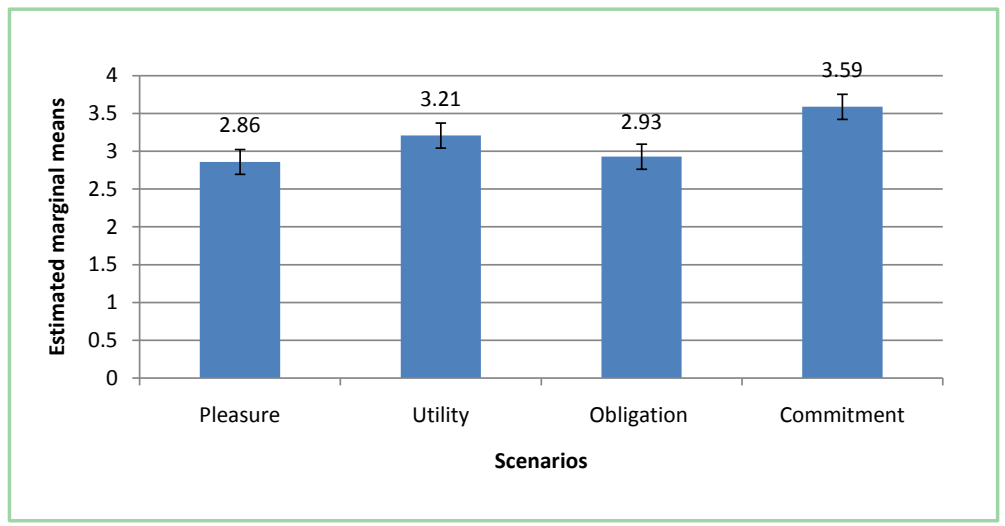

Figure 1. Estimated Marginal Means of Cost Judgments in condition of Resistance.

Secondly, was evaluated the cost factor of the decision for the four levels (scenarios) in the condition of succumbing. Seeing as Mauchly's test was not significant, the condition of sphericity is verified, $\chi^{2}(5)=4.967, p=.420$; epsilon Greenhouser-Geisser $=.933$. The effect of the repeated measurements was significant. $\mathrm{F}(3,600)=6.773 ; p=.000 ; \mathrm{r}=.18 ; \omega^{2}=.02$. Has been then performed the comparisons between the marginal averages with the Sidak correction method (see Figure 2).

The cost of succumbing to an obligation was significantly higher than the cost attributed to succumbing to a choice with an alternative option of pleasure ( $p$ $=.002)$ or utility for the subject $(p=.001)$. The cost of succumbing to a situation of obligation resulted not significantly higher than succumbing to a prior commitment $(p=.159)$. Succumbing to a prior commitment didn't give a significant value higher than the costs recorded for succumbing to pleasure or utility ( $p$ $=.531$ and $p=.280$ ). Succumbing to an obligation presented cost levels higher than those for succumbing to pleasure or utility. To improve the comparison we made a planned comparison adopting the reverse Helmert Contrast method (Difference). The comparison with the previous levels presented a significant $\mathrm{F}$ test: $\mathrm{F}(1,200)=18.735$ with $p=.000$, verifying power equal to .98 (computed using alpha $=.05$ ) and an r-effect size equal to .28 (see Table 4).

Was then evaluated the value factor of the decision for the four levels (scenarios) in the condition of resistance. Seeing as Mauchly's test was significant (Sig. .000), and the condition of sphericity was not verified, $\chi^{2}(5)=23.428$, has been considered the effect of the repeated measurements using the Greenhouse-Geisser correction which resulted significant $\mathrm{F}(2.799,559.81)=3.870 ; p$ $=.01 ; \mathrm{r}=.14 \omega^{2}=.009$. Was then compared the marginal averages using the Sidak correction method (see Figure 3).

The value attributed to a choice of resistance when the alternative option is equally pleasurable was significantly higher (.001) than the other situations in which the alternatives were a utility (.003) and an obligation (.03). (See Table 5) In order to improve the comparison we made a planned comparison adopting the reverse Helmert Contrast method (Difference). The comparison with the previous levels presented a significant $\mathrm{F}$ test. $\mathrm{F}(1,200)=13.614, p$ 
$=.000$, with verifying power equal to .93 (computed using alpha $=.05$ ), and an r-effect size of .24.

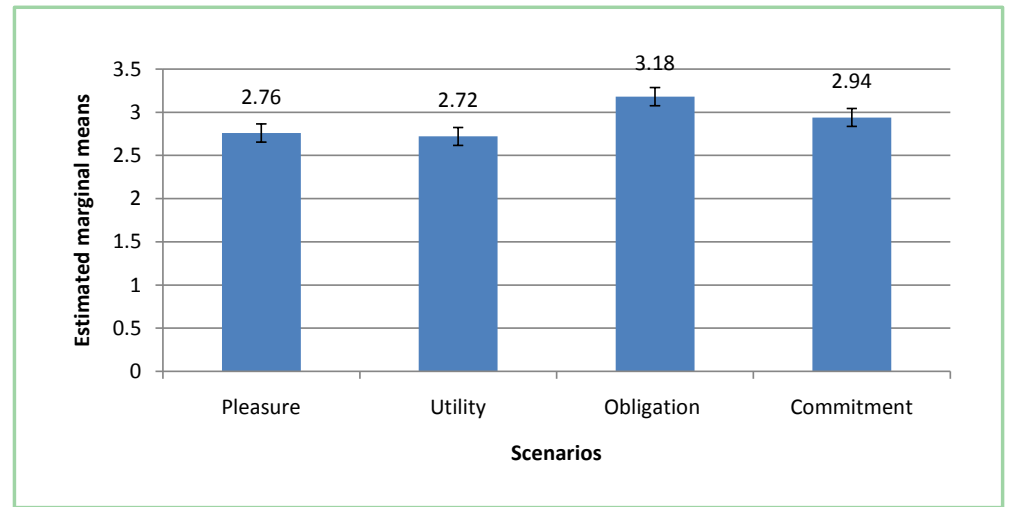

Figure 2. Estimated Marginal Means of Cost Judgments in condition of Succumbing.

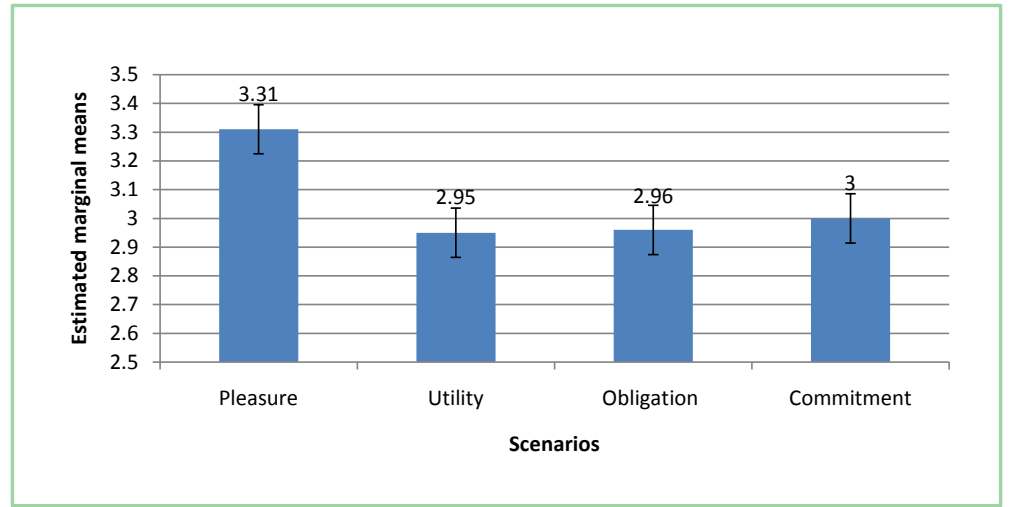

Figure 3. Estimated Marginal Means of Value Judgments in condition of Resistance.

Table 4. Pairwise Comparison of Cost in condition of Succumbing.

\begin{tabular}{|c|c|c|c|c|c|}
\hline (I) & (J) & MD & $\mathrm{SE}$ & $p$ & $\mathrm{BCa} 95 \% \mathrm{CI}$ \\
\hline \multirow{3}{*}{1} & 2 & .040 & .114 & 1.000 & {$[-.26, .34]$} \\
\hline & 3 & $-.428^{*}$ & .118 & .002 & {$[-.74,-.11]$} \\
\hline & 4 & -.189 & .121 & .531 & {$[-.51, .13]$} \\
\hline \multirow{3}{*}{2} & 1 & -.040 & .114 & 1.000 & {$[-.34, .26]$} \\
\hline & 3 & $-.468^{*}$ & .118 & .001 & {$[-.78,-.15]$} \\
\hline & 4 & -.229 & .118 & .280 & {$[-.54, .08]$} \\
\hline \multirow{3}{*}{3} & 1 & $.428^{*}$ & .118 & .002 & {$[.11, .74]$} \\
\hline & 2 & $.468^{*}$ & .118 & .001 & {$[.15, .78]$} \\
\hline & 4 & .239 & .108 & .159 & {$[-.05, .53]$} \\
\hline \multirow{3}{*}{4} & 1 & .189 & .121 & .531 & {$[-.13, .51]$} \\
\hline & 2 & .229 & .118 & .280 & {$[-.08, .54]$} \\
\hline & 3 & -.239 & .108 & .159 & {$[-.53, .05]$} \\
\hline
\end{tabular}

Table legend: $\mathrm{MD}=$ difference of means; $\mathrm{SE}=$ standard error; $\mathrm{CI}=$ confidence interval; $\mathrm{ps}<.05$. 
Table 5. Pairwise Comparison of Value in condition of Resistance.

\begin{tabular}{cccccc}
\hline (I) & $(\mathrm{J})$ & MD & SE & $p$ & BCa 95\% CI \\
\hline & 2 & $.353^{*}$ & .100 & .003 & {$[.08, .62]$} \\
1 & 3 & $.343^{*}$ & .122 & .032 & {$[.02, .67]$} \\
& 4 & .308 & .135 & .131 & {$[-.05, .67]$} \\
& 1 & $-.353^{*}$ & .100 & .003 & {$[-.62,-.09]$} \\
& 3 & -.010 & .119 & 1.000 & {$[-.33, .31]$} \\
& 4 & -.045 & .122 & .999 & {$[-.37, .28]$} \\
& 1 & $-.343^{*}$ & .122 & .032 & {$[-.67,-.02]$} \\
3 & 2 & .010 & .119 & 1.000 & {$[-.31, .33]$} \\
& 4 & -.035 & .125 & 1.000 & {$[-.37, .30]$} \\
& 1 & -.308 & .135 & .131 & {$[-.67, .05]$} \\
4 & 2 & .045 & .122 & .999 & {$[-.28, .37]$} \\
& 3 & .035 & .125 & 1.000 & $.37]$ \\
\hline
\end{tabular}

Table legend: $\mathrm{MD}=$ difference of means; $\mathrm{SE}=$ standard error; $\mathrm{CI}=$ confidence interval; $\mathrm{ps}<.05$.

Was then evaluated the value factor of the decision for the four levels (scenarios) in the condition of succumbing. Since Mauchly's test was not significant (Sig. .196), the condition of sphericity was verified, $\chi^{2}(5)=7.350$. The effect for the repeated measurements was significant. $F(3,600)=8.488$; Sig. $.000 ; r=.20$; $\omega^{2}=.02$. Has been made comparisons between the marginal averages using the Sidak correction method (see Figure 4).

When the subject succumbed to an alternative that was equally pleasurable the value attributed to the choice was significantly less than the other conditions where the subject succumbed to options of utility $(p=.000)$, obligation $(p=.000)$ and prior commitment $(p=.004)$. (See Table 6) To improve the comparison has been made a planned comparison adopting the reverse Helmert contrast (Difference). The comparison with the other levels presented a significant $\mathrm{F}$ test. $\mathrm{F}(1,200)=22.258, p=.000$, with verifying power equal to .99 (computed using alpha $=.05$ ), and an r-effect size of .32 .

Was then evaluated the regret factor of the decision for the four levels (scenarios) in the condition of resistance. Since Mauchly's test was not significant (Sig. .146), the condition of sphericity was verified, $\chi^{2}(5)=8.197$. The effect of the repeated measurements was significant. $\mathrm{F}(3,600)=28.032 ; p=.000 ; \mathrm{r}=.35$; $\omega^{2}=.07$. Has been made comparisons between the marginal averages using the Sidak correction method (see Figure 5).

In the situation of resistance to an alternative, equally pleasurable option, the subject's regret was significantly lower than the other conditions where the subject resisted to the different alternative options of utility $(p=.000)$, obligation $(p=.04)$ and prior commitment $(p=.000)$ (see Table 7).

In the situation of resistance to an alternative option of prior commitment the estimated regret was significantly higher than all the other situa- 
tions of resistance, respectively to pleasure $(p=.000)$, utility $(p=.000)$ and obligation $(p=.000)$.

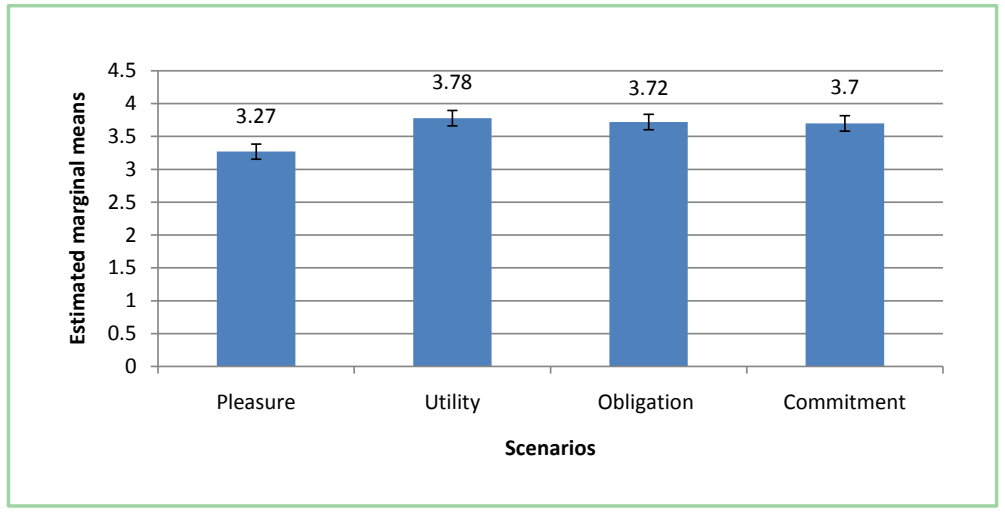

Figure 4. Estimated Marginal Means of Value Judgments in condition of Succumbing.

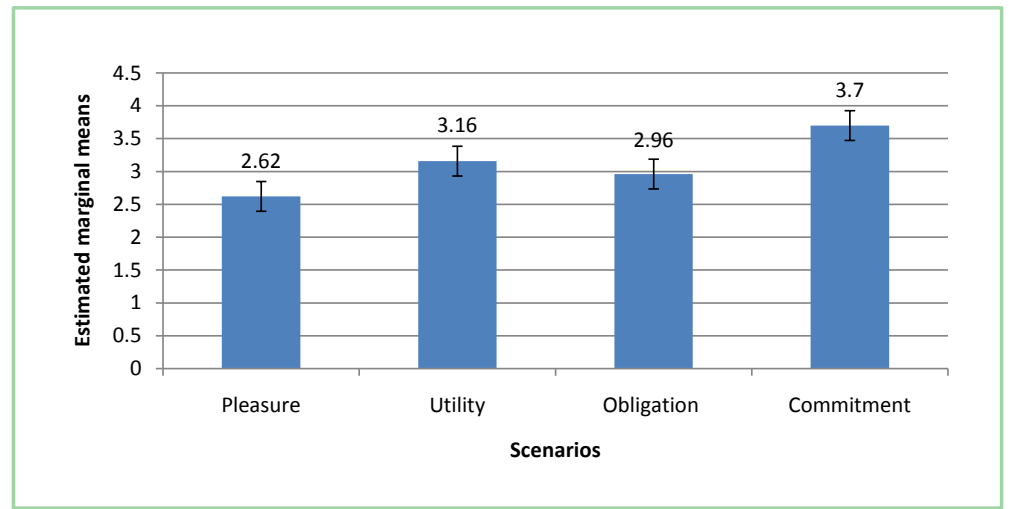

Figure 5. Estimated Marginal Means of Regret Judgments in condition of Resistance.

Table 6. Pairwise Comparison of Value in condition of Succumbing.

\begin{tabular}{cccccc}
\hline (I) & (J) & MD & SE & $p$ & BCa 95\% CI \\
\hline & 2 & $-.512^{*}$ & .115 & .000 & {$[-.82,-.21]$} \\
1 & 3 & $-.453^{*}$ & .111 & .000 & {$[-.75,-.16]$} \\
& 4 & $-.428^{*}$ & .123 & .004 & {$[-.75,-.10]$} \\
& 1 & $.512^{*}$ & .115 & .000 & {$[.21, .82]$} \\
2 & 3 & .060 & .114 & .996 & {$[-.24, .36]$} \\
& 4 & .085 & .106 & .965 & {$[-.20, .37]$} \\
& 1 & $.453^{*}$ & .111 & .000 & {$[.16, .75]$} \\
3 & 2 & -.060 & .114 & .996 & {$[-.36, .24]$} \\
& 4 & .025 & .114 & 1.000 & {$[-.28, .33]$} \\
& 1 & $.428^{*}$ & .123 & .004 & {$[-.10, .75]$} \\
& 2 & -.085 & .106 & .965 & {$[-.33, .28]$} \\
\hline
\end{tabular}

Table legend: $\mathrm{MD}=$ difference of means; $\mathrm{SE}=$ standard error; $\mathrm{CI}=$ confidence interval; $\mathrm{ps}<.05$. 
Table 7. Pairwise Comparison of Regret in condition of Resistance.

\begin{tabular}{|c|c|c|c|c|c|}
\hline (I) & (J) & MD & SE & $p$ & $\mathrm{BCa} 95 \% \mathrm{CI}$ \\
\hline \multirow{3}{*}{1} & 2 & $-.512^{*}$ & .115 & .000 & {$[-.82,-.21]$} \\
\hline & 3 & $-.453^{*}$ & .111 & .000 & {$[-.75,-.16]$} \\
\hline & 4 & $-.428^{*}$ & .123 & .004 & {$[-.75,-.10]$} \\
\hline \multirow{3}{*}{2} & 1 & $.512^{*}$ & .115 & .000 & {$[.21, .82]$} \\
\hline & 3 & .060 & .114 & .996 & {$[-.24, .36]$} \\
\hline & 4 & .085 & .106 & .965 & {$[-.20, .37]$} \\
\hline \multirow{3}{*}{3} & 1 & $.453^{*}$ & .111 & .000 & {$[.16, .75]$} \\
\hline & 2 & -.060 & .114 & .996 & {$[-.36, .24]$} \\
\hline & 4 & .025 & .114 & 1.000 & {$[-.28, .33]$} \\
\hline \multirow{3}{*}{4} & 1 & $.428^{*}$ & .123 & .004 & {$[.10, .75]$} \\
\hline & 2 & -.085 & .106 & .965 & {$[-.37, .20]$} \\
\hline & 3 & -.025 & .114 & 1.000 & {$[-.33, .28]$} \\
\hline
\end{tabular}

Table legend: $\mathrm{MD}=$ difference of means; $\mathrm{SE}=$ standard error; $\mathrm{CI}=$ confidence interval; $\mathrm{ps}<.05$.

To improve the comparison has been made a planned comparison adopting the reverse Helmert Contrast (Difference). The comparison with the other levels presented a significant $\mathrm{F}$ test. $\mathrm{F}(1,200)=59.886, p=.000$, with verifying power equal to 1.00 (computed using alpha $=.05$ ), and an r-effect size of .48 .

Finally was evaluated the regret factor of the decision for the four levels (scenarios) in the condition of succumbing. Since Mauchly's test was not significant (Sig. .421), the condition of sphericity was verified, $\chi^{2}(5)=.975$. The effect of the repeated measurements was significant. $\mathrm{F}(3,600)=5.224 ; p=.001 ; \mathrm{r}=.16 ; \omega^{2}$ $=.01$. Has been made comparisons between the marginal averages using the Sidak correction method (see Figure 6).

In the situation of succumbing to an obligation, the subject's regret was significantly higher than that found in the situations where the subject succumbed to options of equally pleasurable alternative $(p=.002)$, and prior commitment $(p=.02)$. (See Table 8)

To improve the comparison has been made a planned comparison adopting the reverse Helmert Contrast (Difference). The comparison presented a significant $\mathrm{F}$ test. $\mathrm{F}(1,200)=14.250, p=.000$ with verifying power equal to .96 and an r-effect size of .25.

Summarizing the main results above:

- The cost of resistance was maximum when the alternative option was a prior commitment and was significantly high when it presented characteristics of utility for the subject ( $\mathrm{r}$-effect size $=.40$.

- Succumbing to an obligation presented cost levels higher than those for succumbing to pleasure or utility ( $\mathrm{r}$-effect size $=.28$ ).

- The value attributed to a choice of resistance when the alternative option is equally pleasurable was significantly higher than the other situations in which the alternatives were a utility and an obligation $(\mathrm{r}$-effect size $=.24)$. 


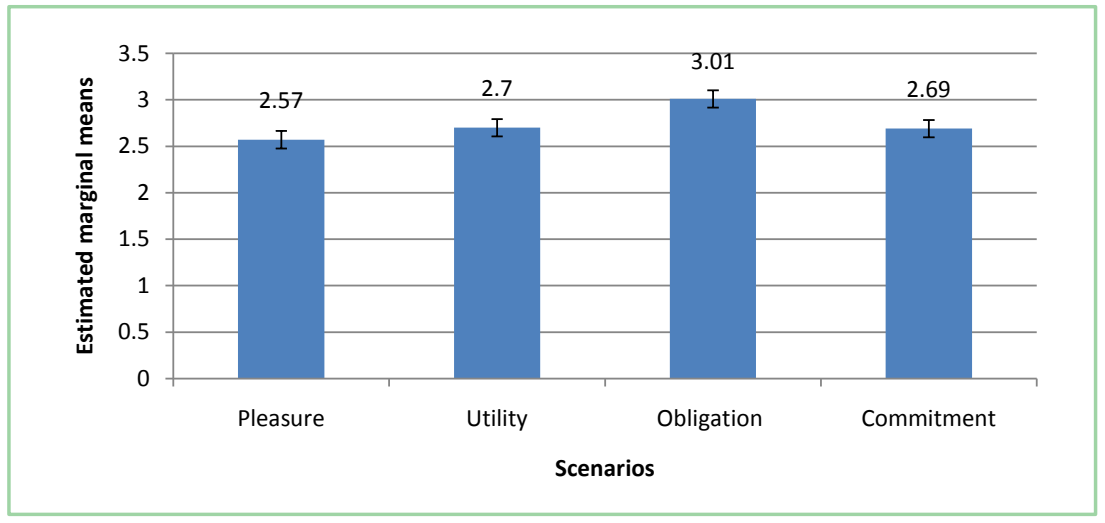

Figure 6. Estimated Marginal Means of Regret Judgments in condition of Succumbing.

Table 8. Pairwise Comparison of Regret in condition of Succumbing.

\begin{tabular}{cccccc}
\hline (I) & $(\mathrm{J})$ & $\mathrm{MD}$ & $\mathrm{SE}$ & $\boldsymbol{p}$ & BCa 95\% CI \\
\hline & 2 & -.134 & .112 & .795 & {$[-.43,-.16]$} \\
1 & 3 & $-.443^{*}$ & .123 & .002 & {$[-.77,-.12]$} \\
& 4 & -.119 & .118 & .896 & {$[-.43, .19]$} \\
& 1 & .134 & .112 & .795 & {$[-.16, .43]$} \\
2 & 3 & -.308 & .121 & .067 & {$[-.63, .01]$} \\
& 4 & .015 & .115 & 1.000 & {$[-.29, .32]$} \\
& 1 & $.443^{*}$ & .123 & .002 & {$[.12, .77]$} \\
3 & 2 & .308 & .121 & .067 & {$[-.01, .63]$} \\
& 4 & $.323^{*}$ & .112 & .025 & {$[.03, .62]$} \\
& 1 & .119 & .118 & .896 & {$[-.19, .43]$} \\
4 & 2 & -.015 & .115 & 1.000 & {$[-.62,-.03]$} \\
& 3 & $-.323^{*}$ & .112 & .025 & \\
\hline
\end{tabular}

Table legend: $\mathrm{MD}=$ difference of means; $\mathrm{SE}=$ standard error; $\mathrm{CI}=$ confidence interval; $\mathrm{ps}<.05$.

- When the subject succumbed to an alternative that was equally pleasurable the value attributed to the choice was significantly less than the other conditions where the subject succumbed to options of utility, obligation and prior commitment $(r$-effect size $=.32)$.

- In the situation of resistance to an alternative option of prior commitment the estimated regret was significantly higher than all the other situations of resistance, respectively to pleasure, utility and obligation ( $r$-effect size $=.48$ ).

- In the situation of succumbing to an obligation, the subject's regret was significantly higher than that found in the situations where the subject succumbed to options of equally pleasurable alternative, and prior commitment $(\mathrm{r}$-effect size $=.25)$.

\section{Discussion}

The analysis compared the post-decisional evaluations between the four scena- 
rios, investigating the conditions of resistance and succumbing separately.

In the condition of resistance the higher cost for the subject was attributed to the renouncement of a utility and prior commitment option. For the latter has been registered the absolute highest cost ever, whereas, whenever the option was a situation that was equally pleasurable or an obligation, the subject evaluated the choice of renouncement by attributing a lower cost to the decision he has made. It is interesting to underline that the resistance to an obligation constituted a relatively easy choice for the subject, especially if we consider how much it would cost him to renounce a personal utility and even more so if he had to derogate an antecedent commitment (Zimmerman, 1986; Sousa, 2009; Vedder, 2019; Tamminga \& Hindriks, 2019).

In the condition of succumbing we found, in regard to the scenario of obligation, a condition that was totally specular to the preceding one, where succumbing to an obligation implied a very high cost for the subject compared to the alternative (pleasurable and/or useful). This confirms the fact that in the confrontation between pleasure and obligation, the attractive force of pleasure manifests all its cogency. So choosing pleasure and therefore resisting an obligation is a much more natural attitude than the contrary. In this case the subject submits to the needs of the obligation with much difficulty and diffidence. Generally speaking, the plea to a moral obligation or an authority does not seem to be the best strategy for inducing subjects to activate themselves and renounce their position of peaceful hedonism and immediate gratification.

As for the value ratings, we can see from the results that there was an interesting specularity in both the conditions of resistance and succumbing when the alternative in question concerned two equally pleasurable conditions. In the case of resistance, the value attributed to this kind of choice was significantly higher than the others (utility, obligation and commitment); while in conditions of succumbing to another pleasurable condition, the value of this kind of choice was considered to be significantly lower than the other three confrontations. This result can be considered substantially in accordance with what has already been elucidated in Brehm's research (1956) and in the numerous successive replicas by other authors (Steele et al., 1993; Lydon, 1999; for a full list see Harmon-Jones \& Mills, 1999). These studies confirmed that after having made a decision, individuals often modify their preferences in order to align them as far as possible with the choice that they have just made. This applies both in conditions of a real choice and in conditions of hypothetical choices, where there are no immediate consequences. This change of preferences has been observed above all in association with two alternatives that were previously considered equivalent (for example two holiday destinations). The classical interpretation of this known paradigm of free choice is that in these conditions the subjects are encouraged by reducing the tension produced by the cognitive dissonance intrinsic in having to choose between two alternatives. We believe that it is possible to hypothesis an alternative mechanism, maybe complementary to the first, according to which the subject in this condition of choice makes an evaluation in 
line with a principle of the kind: "if I have to change, the change must produce an improvement". In this sense, in the first case the value of the resistance consists in having confirmed an antecedent intention, having acknowledged that the alternative would not have caused any further damage (in that it is equivalent). The attribution of value by the subject also "rewards" the intelligence of the choice made. In the second case, the subject, having succumbed to an alternative that is substantially equivalent, makes a negative evaluation thus reducing the value of the choice made and declares the non "advantageousness" and non reasonableness of the same.

As regards the evaluation of regret attributed to the choice made in a condition of resistance, the results showed that a maximum level of regret was reached when the alternative was represented by a prior commitment. This corroborates the fact that despite naturally looking for an immediate gratification from their actions, social commitment represents a very powerful restricting force that, even in conditions of immediate gratification, makes its cogency felt on the subject and activates feelings of distress, guilt and remorse. In the conditions of succumbing the greatest weight of regret is associated to the choice of obligation. Even in this case we can observe that the action orientated to an obligation "weighs" down the subjects not only in terms of cost but also in terms of regret, or rather of a persistent distress regarding the alternative (pleasurable) that he has had to renounce (Connolly et al., 1997; Inman et al., 1997; Zeelenberg et al., 1998; Kivetz \& Simonson, 2002; Connolly \& Zeelenberg, 2002; Mandel, 2003; Guttentag \& Ferrell, 2004; Ksendzova et al., 2015).

\section{Conclusion}

This study has above all demonstrated that the ratings for cost, regret and value associated with a decision can change in the subject according to the subject's awareness of having decided to persevere in his intention of choice while "resisting" another option, or his awareness of having decided to succumb or allow himself to be "tempted" by another option. We also associated quality and structural specificity to this variable in order to highlight the difference between the subjective intentions: a hedonistic choice, a utility choice, a choice conditioned by a social commitment. The comparative analysis (between the scenarios) of the post-decisional evaluation, considered separately in the condition of resistance and in the condition of succumbing, showed that: when persevering in a hedonistic intention, the cost of resistance is greatest when the alternative is a prior commitment made previously by the subject, or is significantly higher when the choice presents characteristics of utility for the subject; it is less when the alternative option is equally pleasurable or when it is an obligation for the subject. On the other hand, when the subject succumbs to an option, keeping faith to an obligation presents a higher level of cost for the subject respect to all the other conditions. The value attributed to a choice of resistance, when the alternative is equally pleasurable, is significantly higher respect to the other situations; whereas when the subject succumbs to an option that is equally pleasura- 
ble, the value attributed to the choice results significantly lowers respect to all the other conditions. In the situation of resistance to an option that foresees keeping a prior commitment, the estimated regret is significantly higher than in all the other situations. In the situation of succumbing the subject's regret is significantly higher whenever the subject decides to renounce a hedonistic choice in the name of an obligation to fulfill. To sum up, the results showed that it does not cost much for subjects to evade an obligation for its own sake or from an authority in the name of a hedonistic choice, while the pressure coming from the thought of having to keep a social commitment is very strong. In general terms, we can deduce that the need to fulfill a moral obligation or an obligation to an authority does not seem to be the best strategy to induce subjects to activate themselves and renounce a position of peaceful hedonism and immediate gratification. This underlines the fact that despite the natural search for immediate gratification from their actions, social commitment represents a very powerful bond for the subjects, even in conditions of immediate gratification, making its cogency felt by activating feelings of regret, guilt and remorse. $s$

Of course this research presents a limitation due to the moderate size of the sample used. We recommend replicating the study with a larger number of subjects. In this way, it would be possible to understand if some of the conclusions of the study can be extended to a more general population. In this research, the comparison between the condition of resistance and that of succumbing has been evaluated as a stimulus able to produce a change of evaluation attitude in the same subject, induced by the alternating identification with a variation of decisional scenarios. A future extension of the study could also consider the idea of assigning different groups of subjects for the two conditions of resistance and succumbing (independent samples). Since the current results have been obtained through the comparison between a pleasant antecedent and decision-making alternatives with different characteristics (utility, obligation, social commitment), there would still be no study extension with the remaining combinations: utility-duty; utility-social commitment; obligation-commitment; including their reversals and comparisons with their counterparts (utility/utility; obligation/duty; social commitment).

\section{Authors' Contribution}

PD, GV, SM designed the study. PD, GV, and SM analyzed the data and discussed the results. PD, GV, and AG drafted the manuscript; SM and AG revised the manuscript. All authors approved the final manuscript. Finally, the authors have agreed to be accountable for all aspects of the manuscript in ensuring that questions related to the accuracy or integrity of any part of it are appropriately investigated and resolved.

\section{Conflicts of Interest}

The authors declare no conflicts of interest regarding the publication of this paper. 


\section{References}

Adams, E., \& Fagot, R. (1959). A Model of Riskless Choice. Behavioral Science, 4, 1-10. https://doi.org/10.1002/bs.3830040102

Baumeister, R. F., \& Heatherton, T. F. (1996). Self-Regulation Failure: An Overview. Psychological Inquiry, 7, 1-15. https://doi.org/10.1207/s15327965pli0701_1

Basu, S., \& Savani, K. (2017). Choosing One at a Time? Presenting Options Simultaneously Helps People Make More Optimal Decisions than Presenting Options Sequentially. Organizational Behavior and Human Decision Processes, 139, 76-91. https://doi.org/10.1016/j.obhdp.2017.01.004

Beike, D. R., Markman, K. D., \& Karadogan, F. (2009). What We Regret Most Are Lost Opportunities: A Theory of Regret Intensity. Personality and Social Psychology Bulletin, 35, 385-397. https://doi.org/10.1177/0146167208328329

Bettman, J. R., Luce, M. F., \& Payne, J. W. (1998). Constructive Consumer Choice Processes. Journal of Consumer Research, 25, 187-121. https://doi.org/10.1086/209535

Brehm, J. W. (1956). Post-Decision Changes in the Desirability of Alternatives. Journal of Abnormal and Social Psychology, 52, 384-389. https://doi.org/10.1037/h0041006

Brehm, J. W., \& Cohen, A. R. (1962). Explorations in Cognitive Dissonance. New York: Wiley. https://doi.org/10.1037/11622-000

Cohen, J. (1988). Statistical Power Analysis for the Behavioral Sciences. Hillsdale, NJ: Lawrence Erlbaum Associates.

Cohen, J. (1992). A Power Primer. Psychological Bulletin, 112, 155-159. https://doi.org/10.1037/0033-2909.112.1.155

Cohen, J. B., \& Goldberg, M. E. (1970). The Dissonance Model in Post-Decision Product Evaluation. Journal of Marketing Research, 7, 315. https://doi.org/10.1177/002224377000700305

Connolly, T., Ordóñez, L. D., \& Coughlan, R. (1997). Regret and Responsibility in the Evaluation of Decision Outcomes. Organizational Behavior and Human Decision Processes, 70, 73-85. https://doi.org/10.1006/obhd.1997.2695

Connolly, T., \& Zeelenberg, M. (2002). Regret in Decision Making. Current Directions in Psychological Science, 11, 212-220. https://doi.org/10.1111/1467-8721.00203

Costanzo, P. J. (2013). Revisiting Cognitive Dissonance Theory: Pre-Decisional Influences and the Relationship to the Consumer Decision-Making Model. Atlantic Marketing Journal, 2, 42-49.

Dhar, R., \& Kim, E. Y. (2007). Seeing the Forest through the Trees: Implications of Construal Level Theory for Consumer Choice. Journal of Consumer Psychology, 17, 96-100. https://doi.org/10.1016/S1057-7408(07)70014-1

Edwards, W., Guttentag, M., \& Snapper, K. (1975). A Decision Theoretic Approach to Evaluation Research. In E. L. Struening, \& M. Guttentag (Eds.), Handbook of Evaluation Research (pp. 139-182). Beverly Hills, CA: Sage.

Ent, M. R., \& Baumeister, R. F. (2014). Embodied Free Will Beliefs: Some Effects of Physical States on Metaphysical Opinions. Consciousness and Cognition, 27, 147-154. https://doi.org/10.1016/j.concog.2014.05.001

Field, A. (2013). Discovering Statistics Using IBM SPSS Statistics. Thousand Oaks, CA: Sage.

Festinger, L. (1957). A Theory of Cognitive Dissonance. Stanford, CA: Stanford University Press.

Festinger, L. (1964). Conflict, Decision, and Dissonance. Stanford, CA: Stanford Univer- 
sity Press.

Fishbach, A., \& Dhar, R. (2005). Goals as Excuses or Guides: The Liberating Effect of Perceived Goal Progress on Choice. Journal of Consumer Research, 32, 370-377. https://doi.org/10.1086/497548

Fishbach, A., Dhar, R., \& Zhang, Y. (2006). Subgoals as Substitutes or Complements: The Role of Goal Accessibility. Journal of Personality and Social Psychology, 91, 232-242. https://doi.org/10.1037/0022-3514.91.2.232

Friedman, E. M. S., Savary, J., \& Dhar, R. (2018). Apples, Oranges, and Erasers: The Effect of Considering Similar versus Dissimilar Alternatives on Purchase Decisions. Journal of Consumer Research, 45, 725-742. https://doi.org/10.1093/jcr/ucy023

Garbarino, E. C., \& Edell, J. A. (1997). Cognitive Effort, Affect, and Choice. Journal of Consumer Research, 24, 147-158. https://doi.org/10.1086/209500

Gilbert, D. T., Morewedge, C. K., Risen, J. L., \& Wilson, T. D. (2004). Looking forward to Looking Backward: The Misprediction of Regret. Psychological Science, 15, 346-350. https://doi.org/10.1111/j.0956-7976.2004.00681.x

Guttentag, R., \& Ferrell, J. (2004). Reality Compared with Its Alternatives: Age Differences in Judgments of Regret and Relief. Developmental Psychology, 40, 764-775. https://doi.org/10.1037/0012-1649.40.5.764

Hammond, J. S., Keeney, R. L., \& Raiffa, H. (1999). Smart Choices: A Practical Guide to Making Better Decisions. Boston, MA: Harvard Business School Press.

Harmon-Jones, E., \& Mills, J. (1999). Cognitive Dissonance: Progress on a Pivotal Theory in Social Psychology. Washington DC: American Psychological Association. https://doi.org/10.1037/10318-000

Higgins, E. T. (2006). Value from Hedonic Experience and Engagement. Psychological Review, 113, 439-460. https://doi.org/10.1037/0033-295X.113.3.439

Hoffmann, V. H., \& Trautmann, T. (2008). Three Types of Impact from the European Emission Trading Scheme: Direct Cost, Indirect Cost and Uncertainty. In B. Hansjurgens, \& R. Antes (Eds.), Economics and Management of Climate Change. Risks, Mitigation and Adaptation (pp. 111-123). New York: Springer. https://doi.org/10.1007/978-0-387-77353-7_9

Hsee, C. K., \& Rottenstreich, Y. (2004). Music, Pandas, and Muggers: On the Affective Psychology of Value. Journal of Experimental Psychology: General, 133, 23-30. https://doi.org/10.1037/0096-3445.133.1.23

Hui, C. M., \& Molden, D. C. (2014). Maintaining Commitment in the Presence of Alternative Opportunities: The Role of Motivations for Prevention or Promotion. Social Cognition, 32, 571-584. https://doi.org/10.1521/soco.2014.32.6.571

Inman, J. J., Dyer, S. S. \& Jia, J. (1997). A Generalized Utility Model of Disappointment and Regret Effects on Post-Choice Valuation. Marketing Science, 16, 97-111. https://doi.org/10.1287/mksc.16.2.97

Iyengar, S. S., \& Lepper, M. R. (2000). When Choice Is Demotivating: Can One Desire Too Much of a Good Thing? Journal of Personality and Social Psychology, 79, 995-1006. https://doi.org/10.1037/0022-3514.79.6.995

Johnson, M. D. (1984). Consumer Choice Strategies for Comparing Noncomparable Alternatives. Journal of Consumer Research, 11, 741-753. https://doi.org/10.1086/209010

Jonker, M. F., Donkers, B., De Bekker-Grob, E. W., \& Stolk, E. A. (2018). Effect of Level Overlap and Color Coding on Attribute Non-Attendance in Discrete Choice Experiments. Value in Health, 21, 767-771. https://doi.org/10.1016/j.jval.2017.10.002

Kahneman, D. (1994). New Challenges to the Rationality Assumption. Journal of Institu- 
tional and Theoretical Economics, 150, 18-36.

Kahneman, D., Knetsch, J. L., \& Thaler, R. (1990). Experimental Tests of the Endowment Effect and the Coase Theorem. Journal of Political Economy, 9, 1325-1348. https://doi.org/10.1086/261737

Keeney, R. L., \& Raiffa, H. (1976). Decisions with Multiple Objectives. New York: Wiley.

Ksendzova, M., Iyer, R., Hill, G., Wojcik, S. P., \& Howell, R. T. (2015). The Portrait of a Hedonist: The Personality and Ethics behind the Value and Maladaptive Pursuit of Pleasure. Personality and Individual Differences, 79, 68-74.

https://doi.org/10.1016/j.paid.2015.01.042

Kivetz, R., \& Simonson, I. (2002). Self Control for the Righteous: Toward a Theory of Precommitment to Indulgence. Journal of Consumer Research, 29, 199-217. https://doi.org/10.1086/341571

Klein, G. A. (1998). Sources of Power: How People Make Decisions. Cambridge, MA: MIT Press.

Krosch, A. R., Figner, B., \& Weber, E. U. (2012) Choice Processes and Their Post-Decisional Consequences in Morally Conflicting Decisions. Judgment and Decision Making, 7, 224-234.

Lydon, J. E. (1999). Commitment and Adversity: A Reciprocal Relation. In W. H. Jones, \& J. M. Adams (Eds.), Handbook of Interpersonal Commitment and Relationship Stability (pp. 193-203). New York: Plenum Press. https://doi.org/10.1007/978-1-4615-4773-0_11

Mandel, D. R. (2003). Counterfactuals, Emotions, and Context. Cognition and Emotion, 17, 139-159. https://doi.org/10.1080/02699930302275

Marcatto, F., \& Ferrante, D. (2012). Regret in Decision Making. Italian Journal of Psychology, 39, 23-47.

Mellers, B., \& Cooke, A. (1994). Tradeoffs Depend on Attribute Range. Journal of Experimental Psychology: Human Perception and Performance, 20, 1055-1067. https://doi.org/10.1037/0096-1523.20.5.1055

Metcalfe, J., \& Mischel, W. (1999). A Hot/Cool System Analysis of Delay of Gratification: Dynamics of Willpower. Psychological Review, 106, 3-19. https://doi.org/10.1037/0033-295X.106.1.3

Park, J. A., Hill, W. T., \& Bonds-Raacke, J. M. (2015). Exploring the Relationship between Cognitive Effort Exertion and Regret in Online vs. Offline Shopping. Computers in Human Behavior, 49, 444-450. https://doi.org/10.1016/j.chb.2015.03.034

Raeva, D., Mittone, L., \& Schwarzbach, J. (2010). Regret Now, Take It Now: On the Role of Experienced Regret on Intertemporal Choice. Journal of Economic Psychology, 31, 634-642. https://doi.org/10.1016/j.joep.2010.04.006

Russo, J. E., \& Dosher, B. (1983). Strategies for Multiattribute Binary Choice. Journal of Experimental Psychology: Learning, Memory, and Cognition, 9, 676-696. https://doi.org/10.1037/0278-7393.9.4.676

Sagi, A., \& Friedland, N. (2007). The Cost of Richness: The Effect of the Size and Diversity of Decision Sets on Post-Decision Regret. Journal of Personality and Social Psychology, 93, 515-524. https://doi.org/10.1037/0022-3514.93.4.515

Salo, I., \& Svenson, O. (2001). Constructive Psychological Processes before and after a Real Life Decision. In C. M. Allwood, \& M. Selart (Eds.), Decision Making: Social and Creative Dimensions (pp. 137-151). Dordrecht: Kluwer.

https://doi.org/10.1007/978-94-015-9827-9_7

Schwartz, B., Ward, A., Monterosso, J., Lyubomirsky, S., White, K., \& Lehman, D. R. 
(2002). Maximizing versus Satisficing: Happiness Is a Matter of Choice. Journal of Personality and Social Psychology, 83, 1178-1197. https://doi.org/10.1037/0022-3514.83.5.1178

Seta, C. E., Seta, J. J., McElroy, G. T., \& Hatz, J. (2008). Regret: The Roles of Consistency-Fit and Counterfactual Salience. Social Cognition, 26, 700-719.

https://doi.org/10.1521/soco.2008.26.6.700

Shamoun, S., \& Svenson, O. (2002). Value Conflict and Post-Decision Consolidation. Scandinavian Journal of Psychology, 43, 325-333. https://doi.org/10.1111/1467-9450.00301

Sharot, T., De Martino, B., \& Dolan, R. (2009). How Choice Reveals and Shapes Expected Hedonic Reaction. Journal of Neuroscience, 29, 3760-3765. https://doi.org/10.1523/JNEUROSCI.4972-08.2009

Sousa, P. (2009). A Cognitive Approach to Moral Responsibility. The Case of a Failed Attempt to Kill. Journal of Cognition and Culture, 9, 171-194. https://doi.org/10.1163/156770909X12489459066183

Steele, C. M., Spencer, S. J., \& Lynch, M. (1993). Self-Image Resilience and Dissonance: The Role of Affirmational Resources. Journal of Personality and Social Psychology, 64, 885-896. https://doi.org/10.1037/0022-3514.64.6.885

Svenson, O., Salo, I., \& Lindholm, T. (2009). Post-Decision Consolidation and Distortion of Facts. Judgment and Decision Making, 4, 397-407.

Svenson, O. (2006). Pre- and Post-Decision Construction of Preferences: Differentiation and Consolidation. In S. Lichtenstein, \& P. Slovic, (Eds.), the Construction of Preference (pp. 356-371). Cambridge: Cambridge University Press. https://doi.org/10.1017/CBO9780511618031.020

Swait, J., \& Adamowicz, W. (2001). The Influence of Task Complexity on Consumer Choice: A Latent Class Model of Decision Strategy Switching. Journal of Consumer Research, 28, 135-148. https://doi.org/10.1086/321952

Tamminga, A., \& Hindriks, F. (2019). The Irreducibility of Collective Obligations. Philosophical Studies, 1-25. https://doi.org/10.1007/s11098-018-01236-2

Trope, Y., \& Liberman, N. (2010). Construal-Level Theory of Psychological Distance. Psychological Review, 117, 440-462. https://doi.org/10.1037/a0018963

Trump, R. K., \& Brucks, M. (2012). Overlap between Mental Representations of Self and Brand. Self Identity, 11, 454-471. https://doi.org/10.1080/15298868.2011.595083

Tversky, A., \& Kahneman, D. (1981). The Framing of Decisions and the Psychology of Choice. Science, 21, 453-458. https://doi.org/10.1126/science.7455683

Van Dijk, E., \& Zeelenberg, M. (2005). On the Psychology of "If Only": Regret and the Comparison between Factual and Counterfactual Outcomes. Organizational Behavior and Human Decision Processes, 97, 152-160. https://doi.org/10.1016/j.obhdp.2005.04.001

Vedder, A. (2019). An Obligation to Enhance? Topoi, 38, 49-52. https://doi.org/10.1007/s11245-017-9483-7

Wilson, T. D., \& Gilbert, D. T. (2005). Affective Forecasting Knowing What to Want. Current Directions in Psychological Science, 14, 131-134. https://doi.org/10.1111/j.0963-7214.2005.00355.x

Zeelenberg, M., van Dijk, W. W., Manstead, A. S. R., \& Van der Pligt, J. (1998). The Experience of Regret and Disappointment. Cognition and Emotion, 12, 221-230. https://doi.org/10.1080/026999398379727

Zeelenberg, M., \& Beattie, J. (1997). Consequences of Regret Aversion: Additional Evi- 
dence for Effects of Feedback on Decision Making. Organizational Behavior and Human Decision Processes, 72, 63-78. https://doi.org/10.1006/obhd.1997.2730

Zeelenberg, M., \& Pieters, R. (2007). A Theory of Regret Regulation 1.0. Journal of Consumer Psychology, 17, 3-18. https://doi.org/10.1207/s15327663jcp1701_3

Zimmerman, B. J. (1986). Development of Self-Regulated Learning: Which Are the Key Subprocesses? Contemporary Educational Psychology, 16, 307-313.

https://doi.org/10.1016/0361-476X(86)90027-5 